sufficient number of patients for a statistically significant result to be possible. Without such a study the precise place of radiology in this poorly understood condition will never be clarified.

\section{Tuberculosis in patients having dialysis}

Patients having regular dialysis treatment have a lowered resistance to infection, ${ }^{1}$ probably owing to a deficiency in cellular immunity. While this may be an advantage when they receive a kidney transplant, they are vulnerable to infection with the tubercle bacillus. Rates of infection up to 16 times that in the normal population have been reported in patients on dialysis, ${ }^{2}$ and in countries where tuberculosis is common in the general population the disease becomes a major problem in dialysis units. For example, 20 of the 329 patients treated by dialysis in Thessaloniki developed tuberculosis. ${ }^{3}$ On the other hand, when the prevalence of tuberculosis in the general population is low infection is correspondingly rare in patients on dialysis. ${ }^{4}$ Even in centres where tuberculosis has been common it has become less so with improved dialysis schedules. $^{2}$

The most common presentation of tuberculosis in patients on dialysis is persistent fever without obvious cause, often accompanied by anorexia, nausea, vomiting, and consequent loss of weight. Similar non-specific symptoms are not uncommon in underdialysed patients, or the fever may be wrongly attributed to pyrogens in the dialyser; all too easily the correct diagnosis may be delayed. Nevertheless, there may be other presenting features, depending on the site of infection. Almost half of all patients have pulmonary or pleural infection or both, and another quarter have tuberculous lymphadenitis, usually cervical. ${ }^{3}$ Tuberculous osteitis is rare, as are miliary infection and meningitis. The symptoms of osteitis may be mistaken for renal osteodystrophy, when again diagnosis will often be delayed.

Even when the diagnosis is suspected clinical confirmation may often prove difficult. The erythrocyte sedimentation rate (ESR) is a useful pointer: though there is some rise in most patients on dialysis, the rise is much more striking in those with tuberculosis. Over three-quarters of the tuberculous patients had ESRs over $100 \mathrm{~mm}$ in one hour in a series reported from Tokyo, ${ }^{2}$ and values around $150 \mathrm{~mm}$ are not uncommon. There is usually a leucocytosis $\left(>10 \times 10^{9} / 1\right)$, and the concentration of $\mathrm{C}$-reactive protein is usually raised; but these tests are non-specific. Unfortunately the reduced cellular immunity usually makes the tuberculin skin test result negative, even in fulminating cases. ${ }^{3}$

A chest radiograph is usually valuable in showing evidence of tuberculous infection, but bacteriological evidence of activity may be difficult to obtain, since culture of the sputum may be sterile and culture of pleural fluid may also be negative. ${ }^{3}$ The diagnosis of tuberculous lymphadenitis is readily made histologically. Tuberculous osteitis is difficult to diagnose: radiological changes occur late, ${ }^{3}$ and the diagnosis usually depends on biopsy or culture of drained pus. Miliary infection may also present difficulties in diagnosis. Changes were seen on the chest radiograph in only one-third of the cases described by Sasaki and his colleagues, ${ }^{2}$ and in two-thirds of their patients diagnosis was made only at necropsy.
Because of the difficulty in establishing the diagnosis bacteriologically and radiologically it may have to be established by a trial of antituberculous treatment ${ }^{2} 3$ in patients with persistent fever, leucocytosis, and raised sedimentation rate in whom other causes of fever have been excluded and in whom there has been no response to broad-spectrum antibiotic treatment.

The drugs used in treating tuberculosis in patients having dialysis are the same as those used in patients with normal renal function, though adjustments are usually necessary in dosage. Streptomycin should be reserved for patients who are acutely ill and need intravenous treatment. The dose is adjusted according to serum concentrations; the drug is removed by dialysis. Isoniazid, which is used in almost all cases, is predominantly eliminated from the body by hepatic acetylation to an inactive metabolite, and the drug and its metabolites are excreted by the kidney. Since acetylator status is not usually assessed the dose of isoniazid in patients having dialysis should not normally exceed $150 \mathrm{mg}$ daily, ${ }^{5}$ though higher doses may be given if serum concentrations can be monitored. Rifampicin is also usually included in treatment. Since it is metabolised by the liver no adjustment of the usual daily dose of 450 to $600 \mathrm{mg}$ (according to body weight) is necessary in renal failure. Ethambutol is usually added to the combination of isoniazid and rifampicin for the first two months of treatment to avoid the emergence of resistant strains. It is excreted largely unchanged by the kidney, and the dose should be reduced to $5 \mathrm{mg} / \mathrm{kg}$ day in patients on dialysis. ${ }^{6}$

In active cases diagnosed bacteriologically, histologically, radiologically, or by an effective trial of treatment, triple treatment with isoniazid, rifampicin, and ethambutol should usually be given for two months, followed by a combination of isoniazid and rifampicin for a further seven months.

Patients with evidence of previous tuberculosis should be given prophylactic chemotherapy with isoniazid as a routine after transplantation, ${ }^{7}$ and this prophylaxis should possibly be extended to patients on dialysis. Such a policy may be worthwhile in dialysis centres where tuberculosis has been a problem, but the case is correspondingly weaker when tuberculosis is rare.

${ }^{1}$ Kaslow RA, Zellner SR. Infection in patients on maintenance haemodialysis. Lancet 1972;ii:117-8.

${ }^{2}$ Sasaki S, Akiba T, Svenaga $M$, et al. Ten years' survey of dialysisassociated tuberculosis. Nephron 1979;24:141-5.

3 Padadimitriou M, Memmos D, Metaxas P. Tuberculosis in patients on regular dialysis. Nephron $1979 ; 24: 53-7$.

4 Frecman RM, Newhouse CE, Lawton RL. Absence of tuberculosis in dialysis patients. FAMA $1975 ; \mathbf{2 3 3}: 1356$.

${ }^{5}$ Fabre J. Balant L, Chavaz A. Recent drug management advances in renal insufficiency. Adv Nephrol $1974 ; 4: 223-48$.

${ }^{6}$ Maher JF. Pharmacologic aspects of regular dialysis treatment. In: Drukker W, Parsons FM, Maher JF, eds. Replacement of renal function in dialysis. The Hague: Nijhoff, 1978:384.

${ }^{7}$ Millar JW, Horne NW. Tuberculosis in immunosuppressed patients. Lancet $1979 ; \mathrm{i}: 1176-8$.

\section{Seasonal cystitis}

Seasonal variations in the incidence of human disease are commonplace, but those in the frequency of urinary tract infections have lacked serious scrutiny. This may be because these infections are endogenous, caused by commensals, so that substantial seasonal variations might not seem very 